\title{
Cognition as a treatment target in depression
}

\author{
M. Kaser ${ }^{1,2,3}$, R. Zaman ${ }^{1}$ and B. J. Sahakian ${ }^{1,2 *}$ \\ ${ }^{1}$ Department of Psychiatry, University of Cambridge, Cambridge, UK \\ ${ }^{2}$ Behavioural and Clinical Neuroscience Institute, Cambridge, UK \\ ${ }^{3}$ Cambridgeshire and Peterborough NHS Foundation Trust
}

Cognitive dysfunction in depression is associated with poorer clinical outcomes and impaired psychosocial functioning. However, most treatments for depression do not specifically target cognition. Neurocognitive deficits such as memory and concentration problems tend to persist after mood symptoms recover. Improving cognition in depression requires a better understanding of brain systems implicated in depression. A comprehensive approach is warranted for refined methods of assessing and treating cognitive dysfunction in depression.

Received 5 October 2016; Revised 25 October 2016; Accepted 31 October 2016; First published online 12 December 2016

Key words: Cognition, depression, neuropsychological model, treatment target.

Depression is a major cause of disability worldwide. It is estimated to be second largest contributor to disease burden by 2020 according to World Health Organization (World Federation of Mental Health, 2012). Depression could interfere with various aspects of functioning including work, quality of life, or psychosocial functioning. At workplace, depression is one of the main causes of absenteeism and presenteeism (Druss et al. 2001). Absenteeism refers to direct impact of depression on occupational functioning where people cannot attend work due to depressive illness. Presenteeism is related to decreased performance at work when people still attend to work despite ongoing illness or they cannot return to previous performance levels after recovery. Cognitive symptoms were suggested to be major factor contributing to functional impairments (McIntyre et al. 2013).

Clinically, cognitive symptoms are commonly endorsed by the patients both during episodes and as residual symptoms (Conradi et al. 2011). Numerous studies reported poorer neuropsychological performance in tests of memory, attention and executive function in patients with depression (Bora et al. 2013). Cognitive dysfunction in depression is associated with poorer psychosocial functioning (Jaeger et al. 2006), and higher rate of relapse (Majer et al. 2004). Memory and concentration problems in depression are linked to unemployment or work impairment (Buist-Bouwman et al. 2004; Lam et al. 2012). The impact of cognitive dysfunction on functioning may be related to persistence of cognitive deficits even

\footnotetext{
* Address for correspondence: Professor B. J. Sahakian, University of Cambridge, Cambridge, UK.

(Email: bjs1001@medschl.cam.ac.uk)
}

after mood symptoms recover. A meta-analysis of studies using a standardized test battery (CANTAB) showed that the magnitude of cognitive deficits during episode and in remission were comparable (Rock et al. 2014). On the other hand, recurrent nature of depressive disorders and accompanying cognitive dysfunction could contribute to further disability associated with depression. The 'toxicity' of repeated episodes (Gorwood et al. 2008) on cognition, also known as 'scarring' (Kessing, 1998) was proposed to explain persistence of cognitive dysfunction. According to this, each episode leads to accumulation of vulnerability, hence leading to further decline in cognitive functioning over the years. In later life, memory deficits seen in depression could be vulnerability factors for dementia (Kessing \& Andersen, 2004). In a longitudinal epidemiological study (Baltimore Longitudinal Study of Aging), elevated depressive symptoms over the life course were associated with increased risk of dementia (Dotson et al. 2010).

Despite the clear impact on the course of depression, cognition is yet to be accepted as a treatment target for depression. In a recent survey, more than $90 \%$ of the patients with a history of depression reported significant impact of cognitive problems in their daily living activities. However, only 50\% have ever been asked about cognitive dysfunction by a healthcare professional (Clark Health Communications, 2015). Most currently available treatments were shown to be effective for overall mood symptoms, whereas the effects on cognitive functions were rarely tested in double-blind placebo controlled trials. The scarcity of evidence on medication effects on cognitive functions in depression makes it difficult to draw conclusions. A comprehensive review on previous studies highlighted the problems with existing research. Mainly, assessment 
methods and clinical features of study populations varied markedly. Most of the studies were not placebo controlled, and focus was heavily on depression in older adults. Overall, conventional antidepressants have very limited, if any effects at all on cognition in depression (Keefe et al. 2014). In a recent randomized longitudinal study, it was shown that 8-week treatment with escitalopram, sertraline, or venlafaxine did not have significant effects on cognitive functions as measured by a comprehensive neuropsychological battery. This applied to patients in the same sample who have achieved clinical remission with the treatment (Shilyansky et al. 2016). The persistence of cognitive dysfunction even after mood symptoms recover has been well documented in previous studies (Hasselbalch et al. 2011) which suggested that cognitive functions were not addressed by medication. However, recently licensed antidepressant vortioxetine stands out among other antidepressants as it was shown to improve cognitive functions. The first study to report pro-cognitive effects of vortioxetine showed improvement in digit symbol test performance in elderly depressed patients (Katona et al. 2012). Consistent with the initial findings, a large randomized controlled trial showed that vortioxetine improved cognitive functions in non-elderly patients with depression (McIntyre et al. 2014). Multiple regression analyses revealed that there was a direct effect on cognition independent from the effects on mood. It should be noted that effect sizes for most tests except digit symbol substitution test were small in this study and replication is needed. Vortioxetine is a multi-modal pharmacological agent acting on a number of neurotransmitters including dopamine, noradrenaline, and diverse actions on different serotonin receptors. It was suggested that the pro-cognitive effect of vortioxetine might be associated with its stimulatory effects if $5-\mathrm{HT}_{1 \mathrm{~A}}$ receptors and its inhibitory effects on $5-\mathrm{HT}_{3}$. Both mechanisms enhance cortical glutamatergic transmission (Mørk et al. 2012). Another noteworthy medication is modafinil which was shown to improve cognition in depression as adjunctive treatment (DeBattista et al. 2004). One of the proposed mechanisms of modafinil's pro-cognitive effect is through augmenting glutamatergic transmission in hippocampus (Scoriels et al. 2013). Modafinil augmentation showed beneficial effects on mood and fatigue (Goss et al. 2013) which may make its use more favourable for patients with persistent cognitive dysfunction.

There is suggestion that monoaminergic antidepressants may have favourable effects on hot (emotionladen) cognition (Roiser et al. 2012). Antidepressants were shown to alter response biases to emotional stimuli very early in the course of treatment (Harmer et al. 2009). The mechanism linking this effect to therapeutic effects is yet to be elucidated. The interplay between negative emotional biases and cognitive functions in depression has been formulated in cognitive neuropsychological model. According to this model proposed by Roiser et al. (2012, fig. 1), bottom-up affective biases and top-down attentional biases (due to dysfunctional cognitive control) help to maintain the depressive state. Pharmacological interventions (i.e. antidepressant medication) alter the affective processing of emotional stimuli, thus helping to reverse negative biases and eventually leading to remission of mood symptoms. Within this model, psychological treatments are suggested to work towards gaining cognitive control over negative biases. Cognitive neuropsychological model could help guiding the research to identify treatments specifically addressing cognition.

The challenges and potential research areas into cognitive dysfunction in depression were published in a report by National Academy of Sciences (National Academies of Sciences, Engineering, and Medicine, 2015). It is highlighted that one of the main points regarding research into new interventions is the need for change in trial designs to include cognitive outcomes. Regulators such as Food and Drug Administration in the USA hold the expectations that interventions should help people to function better in work and daily living activities, therefore psychosocial functioning measures are equally important. With the developing mobile technologies and more accessible neurocognitive testing methods, future studies will be able to provide the much needed information on cognitive functioning in depression. New trial designs could be utilized to test the promising interventions for improving cognition in depression.

In summary, cognitive dysfunction in depression is a significant aspect of depression. It is associated with poorer functioning, poses risk for relapse and persists even after mood symptoms recover. A more comprehensive and refined approach is warranted to address the unmet need for improving cognitive functions in depression. In our opinion cognition is a key target for treatment in depression.

\section{Declaration of Interest}

B.J.S. consults for Cambridge Cognition and Mundipharma. She has consulted for Lundbeck.

\section{References}

Bora E, Harrison BJ, Yücel M, Pantelis C (2013). Cognitive impairment in euthymic major depressive disorder: a meta-analysis. Psychological Medicine 43, 2017-2026.

Buist-Bouwman MA, Ormel J, de Graaf R, Vollebergh WA (2004). Functioning after a major depressive episode: 
complete or incomplete recovery? Journal of Affective Disorders 82, 363-371.

Clark Health Communications (2015). Survey of British adults diagnosed with depression on behalf of Clark Health Communications (http://www.comres.co.uk/wp-content/ uploads/2015/09/Clark-Health-Communications_ Cognitive-Dysfunction-in-Depression.pdf).

Conradi HJ, Ormel J, De Jonge P (2011). Presence of individual (residual) symptoms during depressive episodes and periods of remission: a 3-year prospective study. Psychological Medicine 41, 1165-1174.

DeBattista C, Lembke A, Solvason HB, Ghebremichael R, Poirier J (2004). A prospective trial of modafinil as an adjunctive treatment of major depression. Journal of Clinical Psychopharmacology 24, 87-90.

Dotson VM, Beydoun MA, Zonderman AB (2010). Recurrent depressive symptoms and the incidence of dementia and mild cognitive impairment. Neurology 75, 27-34.

Druss BG, Schlesinger M, Allen Jr. HM (2001). Depressive symptoms, satisfaction with health care, and 2-year work outcomes in an employed population. American Journal of Psychiatry 158, 731-734.

Gorwood P, Corruble E, Falissard B, Goodwin GM (2008). Toxic effects of depression on brain function: impairment of delayed recall and the cumulative length of depressive disorder in a large sample of depressed outpatients. American Journal of Psychiatry 165, 731-739.

Goss AJ, Kaser M, Costafreda SG, Sahakian BJ, Fu CH (2013). Modafinil augmentation therapy in unipolar and bipolar depression: a systematic review and meta-analysis of randomized controlled trials. Journal of Clinical Psychiatry 74, 1101-1107.

Harmer CJ, O'Sullivan U, Favaron E, Massey-Chase R, Ayres R, Reinecke A, Goodwin GM, Cowen PJ (2009). Effect of acute antidepressant administration on negative affective bias in depressed patients. American Journal of Psychiatry 166, 1178-1184.

Hasselbalch BJ, Knorr U, Kessing LV (2011). Cognitive impairment in the remitted state of unipolar depressive disorder: a systematic review. Journal of Affective Disorders 134, 20-31.

Jaeger J, Berns S, Uzelac S, Davis-Conway S (2006). Neurocognitive deficits and disability in major depressive disorder. Psychiatry Research 145, 39-48.

Katona C, Hansen T, Olsen CK (2012). A randomized, double-blind, placebo-controlled, duloxetine-referenced, fixed-dose study comparing the efficacy and safety of $\mathrm{Lu}$ AA21004 in elderly patients with major depressive disorder. International Clinical Psychopharmacology 27, 215-223.

Keefe RS, McClintock SM, Roth RM, Doraiswamy PM, Tiger S, Madhoo M (2014). Cognitive effects of pharmacotherapy for major depressive disorder: a systematic review. Journal of Clinical Psychiatry 75, 864-876.
Kessing LV (1998). Cognitive impairment in the euthymic phase of affective disorder. Psychological Medicine 28, 1027-1038.

Kessing LV, Andersen PK (2004). Does the risk of developing dementia increase with the number of episodes in patients with depressive disorder and in patients with bipolar disorder? Journal of Neurology, Neurosurgery and Psychiatry 75, 1662-1666.

Lam RW, Michalak EE, Bond DJ, Tam EM, Axler A, Yatham LN (2012). Which depressive symptoms and medication side effects are perceived by patients as interfering most with occupational functioning? Depression Research and Treatment. Article ID 630206, 6 pp, doi:10.1155/2012/630206.

Majer M, Ising M, Künzel H, Binder EB, Holsboer F, Modell S, Zihl J (2004). Impaired divided attention predicts delayed response and risk to relapse in subjects with depressive disorders. Psychological Medicine 34, 1453-1463.

McIntyre RS, Cha DS, Soczynska JK, Woldeyohannes HO, Gallaugher LA, Kudlow P, Alsuwaidan M, Baskaran A (2013). Cognitive deficits and functional outcomes in major depressive disorder: determinants, substrates, and treatment interventions. Depression and Anxiety 30, 515-527.

McIntyre RS, Lophaven S, Olsen CK (2014). A randomized, double-blind, placebo-controlled study of vortioxetine on cognitive function in depressed adults. International Journal of Neuropsychopharmacology 17, 1557-1567.

Mørk A, Pehrson A, Brennum LT, Nielsen SM, Zhong H, Lassen AB, Miller S, Westrich L, Boyle NJ, Sanchez C, Fischer CW (2012). Pharmacological effects of Lu AA21004: a novel multimodal compound for the treatment of major depressive disorder. Journal of Pharmacology and Experimental Therapeutics 340, 666-675.

National Academies of Sciences, Engineering, and Medicine (2015). Enabling Discovery, Development, and Translation Of treatments for Cognitive Dysfunction in Depression: Workshop Summary. National Academies Press: Washington, DC.

Rock PL, Roiser JP, Riedel WJ, Blackwell AD (2014). Cognitive impairment in depression: a systematic review and meta-analysis. Psychological Medicine 29, 1-12.

Roiser JP, Elliott R, Sahakian BJ (2012). Cognitive mechanisms of treatment in depression. Neuropsychopharmacology 371, 117-136.

Scoriels L, Jones PB, Sahakian BJ (2013). Modafinil effects on cognition and emotion in schizophrenia and its neurochemical modulation in the brain. Neuropharmacology 64, 168-184.

Shilyansky C, Williams LM, Gyurak A, Harris A, Usherwood T, Etkin A (2016). Effect of antidepressant treatment on cognitive impairments associated with depression: a randomised longitudinal study. Lancet Psychiatry 3, 425-435.

World Federation of Mental Health (2012). Depression: a global crisis (http://www.who.int/mental_health/ management/depression/who_paper_depression_wfmh_ 2012.pdf?ua=1). 